\title{
Relaciones entre la teoría pura del derecho y los derechos humanos
}

\author{
¿Contradicción o compatibilidad?
}

Por Sisti Pedro

Sumario: 1.Introducción. 2- Clasificación de tipo de Positivismo Jurídico al que abreva la Teoría Pura del Derecho en la Doctrina. 3. Los Derechos Humanos y su fundamento iusfilosófico. 4. Primer análisis de compatibilidad: los Dualismos del Derecho a la luz de los Derechos Humanos. 5. Segundo análisis de compatibilidad: la Grundnorm (Norma Fundamental) y los Derechos Humanos. 6. Reflexión Final. 7. Bibliografía

\begin{abstract}
El trabajo pretende mostrar un aspecto no abordado usualmente de la Teoría Pura del Derecho de Kelsen: su compatibilidad (o no) con el desarrollo actual del Derecho Internacional de los Derechos Humanos. Se analizará lo dicho por la doctrina acerca de la Teoría Pura y de la ubicación filosófica de los Derechos Humanos en el iuspositivismo o el iusnaturalismo, para luego establecer los puntos de partida que se tomarán al momento de compatibilizar la Teoría Pura con esta rama del derecho. En particular se analizarán de la Teoría Pura las cuestiones de los Dualismos del Derecho y la Grundnorm (Norma Fundamental), para ver si la teoría kelseniana puede ser compatible o no con relación a los Derechos Humanos.

Palabras claves: Filosofía del Derecho/ Derechos Humanos/ Teoría General del Derecho/ Positivismo Jurídico/ Kelsen.
\end{abstract}

\section{Introducción}

En este trabajo abordaremos la relación entre la Teoría Pura del Derecho de Hans Kelsen y los Derechos Humanos, que a simple vista pueden parecer alejadas o incluso contradictorias, pero conforme se irá desarrollando, se verá que existe la posibilidad cierta no solo de compatibilizar el desarrollo actual del Derecho Internacional de los Derechos Humanos (en adelante DDHH) con la Teoría Pura del Derecho (en adelante TPD), sino que incluso puede servir para abrir la discusión acerca de la justificación última de los sistemas normativos locales y su relación con el Derecho Internacional en general, y con los DDHH en particular, algo que por la última jurisprudencia de nuestra Corte Suprema de Justicia ha vuelto a entrar en discusión. 
Sin dudas la TPD de Hans Kelsen ha sido uno de los libros más analizados desde su publicación en el marco de la Teoría del Derecho ${ }^{1}$. Sin embargo, este trabajo pretende esbozar una aproximación a una cuestión no tan analizada: la compatibilidad (o no) de la TPD con los DDHH. Algo que a primera vista parecería indudablemente contradictorio.

Por ser la TPD un libro tan analizado, tendremos que proceder a deconstruir ciertas elaboraciones de otros autores que se han realizado sobre la misma, en las cuales muchas veces se ha desnaturalizado o malinterpretado lo dicho por el autor. También abordaremos algunas posturas iusfilosóficas acerca de los DDHH, que debemos adelantar que han sido identificados tanto con el iusnautralismo como con el iuspositivismo, lo cual demuestra que no es una cuestión saldada.

Luego nos adentraremos en lo que resultará ser la cuestión central de este trabajo: analizar la compatibilidad (o no) de la TPD con los DDHH, tomando específicamente dos cuestiones centrales desarrolladas por Kelsen: los Dualismos del Derecho y la Norma Fundamental (esta última probablemente sea la cuestión más criticada y malinterpretada de toda la teoría kelseniana).

Por último, haremos algunas reflexiones acerca de las conclusiones a las cuales arribamos y proyectaremos las posibles consecuencias de las mismas en torno a la justificación de los ordenamientos jurídicos nacionales.

\section{2- Clasificación de tipo de Positivismo Jurídico al que abreva la Teoría Pura del Derecho en la Doctrina}

Existe gran consenso respecto de que Kelsen, y especialmente su TPD, es uno de los hitos más importantes (si no el más) en lo que hace al estudio del derecho en el Siglo XX. Han partido de la TPD, ya sea para atacarla, defenderla o complementarla, autores internacionales de la talla de: Hart, Ross, Dworkin, Rawls, Bobbio, Ferrajoli, Alexy, Atienza y Guastini entre muchos otros. De más está decir que en nuestro país la influencia de Kelsen (de la mano de Cossio principalmente) ha sido muy profunda, han dedicado largas páginas a su estudio autores como: Cossio, Carrió, Alchourrón, Bulygin, Vernengo, Nino, Smith y tantos otros.

Es indudable que intentar escribir algo novedoso acerca de la TPD resulta altamente dificultoso, si es que no imposible. Pero justamente por haber sido tan estudiada e interpretada, es que cobra una mayor importancia delimitar cuáles de las posturas atribuidas al autor son acertadas, y cuáles son una desviación de lo establecido en el texto.

1 Adoptando el criterio clasificatorio atribuido a Bobbio. Guastini, Riccardo. Distinguiendo. Traducción de Jordi Ferrer i Beltrán. Editorial Gedisa, Barcelona. 
Lo primero que debe establecerse al hablar de la TPD, es identificar qué edición (y traducción) se va a tomar como fuente, ya que incluso existen trabajos acerca de si su Teoría era la misma o cambió sustancialmente entre la primera y la segunda edición ${ }^{2}$.

En respuesta a este primer planteo, se aclara que para este trabajo hemos decidido tomar como fuente de interpretación tanto la primer edición publicada por EUDEBA (traducida del alemán al francés, y de este luego al español), como la segunda edición publicada por la UNAM (traducida directamente del alemán por Vernengo). Lo que haremos será, en los temas específicos, mencionar lo que dice una y otra edición, intentando obtener una postura uniforme.

Una vez establecidas las fuentes de interpretación, nos queda analizar lo que la doctrina ha dicho respecto de la TPD. No hace falta aclarar que esta cuestión en particular no podrá abarcarse de manera total en este trabajo, de hecho ni siquiera con un grupo de investigación destinado pura y exclusivamente a esta tarea durante años podría asegurarse que se ha estudiado en su totalidad lo que la doctrina ha dicho acerca de la TPD. Hecha esta salvedad, abordaremos en este apartado lo que la doctrina dijo de esta teoría respecto de su ubicación iusfilosófica como positivismo jurídico (algo en general no cuestionado, pero que tiene sus matices); en otro apartado analizaremos específicamente lo que la doctrina ha dicho acerca de la Grundnorm (Norma Fundamental), que sin dudas es una de las cuestiones que más se ha criticado y desinterpretado de la teoría kelseniana.

Lo primero que debemos decir es que dentro del positivismo jurídico, se han englobado teorías (y teóricos) muy diferentes. Para tener una idea, se identifican como positivistas a los siguientes autores: Austin, Bentham, Kelsen, Hart, Bobbio, Ross, Carrió, Alchourrón, Bulygin y Ferrajoli. Por lo tanto, debemos establecer a qué nos referimos cuando hablamos de positivismo. Una de las mejores maneras de hacer esto, es clasificar distintos tipos de positivismo.

En la doctrina se han realizado diferentes clasificaciones del positivismo jurídico. Una de las más conocidas es la de Bobbio (1997), que divide al positivismo en tres, de acuerdo a la "intensidad" o el grado de positivismo: el primero, y menos intenso, como una forma de acercarse al derecho (que llamaremos metódico o enfoque); el segundo en un término medio, como una teoría o concepción del derecho (teórico); y, el tercero y más intenso, como una determinada ideología de la justicia (ideológico).

Nino, tomando como punto de partida la clasificación de Bobbio (y sumando aportes de Carrió y Ross), ha hecho una clasificación de cuatro tipo de positivismos jurídicos: el escepticismo ético; el formalismo jurídico; el positivismo ideológico (o pseudopositivismo según Ross); y el positivismo conceptual (Nino, 2007: 157). En esta última quedarían englobados todos los teóricos que él identifica como positivistas. Para Nino (2007), el positivismo conceptual "expresa que el sistema legal de una sociedad puede ser

2 Han escrito al respecto Mario G. Losano (La Teoría Pura del Derecho: del Logicismo al Irracionalismo) y también Stanley L. Paulson (El período posterior a 1960 de Kelsen: ¿Ruptura o Continuidad?), por mencionar algunos. 
identificado y descripto sobre la base de circunstancias fácticas y sin necesidad de evaluar su coincidencia o no con principios de justicia y moralidad social”.

Atienza por su lado no comparte la postura de Nino de que el formalismo jurídico sea un tipo de positivismo jurídico, y lo separa de este. Dentro del positivismo normativista identifica a dos vertientes: la de Kelsen que es más radical y considera al Derecho como un orden de normas coactivas; y la de Hart, que Atienza (2014) la considera más moderada y sofisticada, para quien el Derecho está compuesto por normas primarias y secundarias (estas últimas no son coactivas, sino que se refieren a las primarias)

Dentro de estas clasificaciones, podríamos decir sin ningún lugar a dudas que podemos trazar un cierto paralelismo entre el Positivismo Teórico (de Bobbio) y el Positivismo Conceptual (de Nino), ubicando indudablemente a Kelsen en ambas posturas. En adelante, cuando hablemos de Positivismo, lo haremos desde esta mirada.

\section{Los Derechos Humanos y su fundamento iusfilosófico}

En este acápite no nos preocuparemos tanto acerca de la definición de los DDHH, qué derechos protegen y dónde podemos encontrarlos, sino que nos adentraremos en la discusión acerca de si los DDHH tienen una justificación iusfilosófica que se encuadra mejor en el iuspositivismo o en el iusnaturalismo.

Para dejar en claro, tomamos una postura amplia de DDHH, incluyendo todo tipo de instrumentos internacionales. Identificamos el inicio de los DDHH en 1948, con la sanción de la Declaración Americana de Derechos y Deberes del Hombre en el ámbito de la Organización de Estados Americanos y también con la sanción de la Declaración Universal de Derechos Humanos en el ámbito de Naciones Unidas. Englobando luego a la totalidad de Pactos, Tratados y Convenciones dictadas, como así también las decisiones y recomendaciones elaboradas por los organismos creados para su control (Comisión Interamericana, Comités de Naciones Unidas, Corte Interamericana, Relatores, Grupos de Trabajo, etc.).

De más está decir que se han elaborado diferentes tipos de justificaciones filosóficas acerca de lo DDHH, como bien dice Spector (2001: 34): “...las teorías disponibles recorren todo el espectro de la filosofía moral”. Realizando un excesivo recorte (necesario por las características de este trabajo), podemos establecer que una de las teorías centrales en esta cuestión ha sostenido que los DDHH pertenecen a una corriente iusnaturalista, porque se ha entendido que los mismos entran dentro de lo que se ha denominado en la doctrina anglosajona "Derechos Morales", que existen más allá (y antes) de su consagración normativa. Por ende, los DDHH son una expresión de la moral que sirve de justificación última al Derecho. Avalando esta postura podemos encontrar a autores como Nino y Dworkin.

Encontramos críticas muy interesantes a esta fundamentación moral de los DDHH, como por ejemplo la realizada por Vernengo, cuando sostiene que a dicha fundamentación la considera "infructuosa teóricamente y prácticamente riesgosa como 
política”, para luego aseverar que los DDHH "tiene que ser vistos como manifestaciones de esa libertad metafísica, como modos de la voluntad de poder" (Vernengo, 1990:298).

También elaboró una crítica a esta postura Bulygin (1987: 84), cuando manifestó que

la fundamentación de los derechos humanos en el derecho natural o en una moral absoluta no sólo es teóricamente poco convincente, sino políticamente sospechosa, pues una fundamentación de ese tipo tiende a crea una falsa sensación de seguridad: si los derechos humanos tienen una base tan firme, no hace falta preocuparse mayormente por su suerte, ya que ellos no pueden ser aniquilados por el hombre.

Por otro lado, existe la postura de que los DDHH se encuentran en el ámbito del Derecho Positivo, y por lo tanto dependen de su consagración. En esta corriente podemos encontrar distintas posturas: por un lado, las que sostienen que la consagración en cualquier ámbito (nacional o internacional) es suficiente para tomarlos como derecho positivo; por otro lado, quienes entienden que es necesaria la recepción en el derecho interno para que cobren vigencia efectiva. Estas diferencias no las abordaremos en este trabajo apartado, ya que al desarrollar la cuestión de la Norma Fundamental, las abordaremos, baste con decir que abrevamos a la primer postura, en la cual ya sea que se consagre en el ámbito internacional o nacional, deviene su obligatoriedad.

Como corolario de este apartado, creemos que si bien resulta atractiva la postura de entender que los DDHH forman parte de una moral universal (ya sea absoluta o variable), por entender que les brinda mayor legitimidad, preferimos adherir a la postura positivista y hacer nuestros los argumentos claramente planteados por Bulygin: "Para la concepción positivista, en cambio, los derechos humanos son una muy frágil, pero no por ello menos valiosa conquista del hombre, a la que hay que cuidar con especial esmero, si no se quiere que esa conquista se pierda, como tantas otras" ${ }^{3}$. De hecho, incluso algunos autores que han defendido la postura iusnaturalista de los DDHH, sostienen que estos tienen una "pretensión de positivización" (Spector, 2001: 45), lo cual demuestra que ambas teorías no se encuentran tan alejadas.

Una mención especial debemos hacer a la muy interesante y novedosa teoría elaborada por Barberis (2013) , cuyas palabras finales citamos por parecernos una de las más apropiadas para tomar como el adecuado enfoque de este trabajo:

Dejando de lado las viejas concepciones iusnaturalistas y positivistas estándar, este ensayo ha propuesto adoptar una concepción positivista refinada que puede calificarse de evolucionista, especificacionista y pluralista. Evolucionista: los derechos humanos, como todos los valores, son agregaciones de evaluaciones subjetivas, que pueden ser institucionalizadas jurídicamente y objetivamente por las constituciones.

3 Bulygin, Eugenio, obra citada. 
Especificacionista: los derechos humanos son declarados por principios genéricos que admiten especificaciones diferentes. Pluralista: los derechos humanos son valores plurales y siempre virtualmente conflictivos, y para solucionar el conflicto necesitan técnicas como la ponderación y el juicio de proporcionalidad. Estas concepciones son realistas y no optimistas, pero precisamente por esta razón no debilitan sino fortalecen la lucha para convertir los derechos en adquisiciones evolutivas de la especie humana (40).

\section{Primer análisis de compatibilidad: los Dualismos del Derecho a la luz de los Derechos Humanos}

En la TPD, el Dualismo madre o central es sin dudas el Dualismo: Derecho PositivoDerecho Natural. También identificado como Dualismo Ontológico. De alguna manera hemos abordado este Dualismo en el anterior acápite, cuando nos preguntamos acerca del fundamento filosófico de los DDHH. Si hubiéramos entendido que la fundamentación era iusnaturalista, nos hubiera resultado imposible abordar las cuestiones que analizaremos en este acápite y el anterior, ya que para Kelsen el Derecho Natural no es (ni puede ser) el fundamento lógico del Derecho Positivo. Esta es otra razón para sostener la postura que considera que los DDHH son parte del Derecho Positivo.

A continuación del Dualismo Ontológico, nos encontramos con los Dualismos Metodológicos, que son: Derecho Objetivo-Derecho Subjetivo; Persona Física-Persona Jurídica; Derechos Reales-Derechos Personales; Derecho Público-Derecho Privado; Derecho-Estado; Derecho Nacional-Derecho Internacional ${ }^{4}$. Estos Dualismos Kelsen los unifica, demostrando que en realidad no son dos cosas (normatividades) diferentes, sino que son una única y misma cosa: Derecho Positivo.

En este acápite abordaremos los primeros dos Dualismos Metodológicos, es decir: Derecho Objetivo-Derecho Subjetivo y Persona Física-Persona Jurídica. Ya que tanto los Dualismos acerca de los Derechos Reales-Derechos Personales, el de Derecho PúblicoDerecho Privado, como también el de Derecho-Estado han sido indudablemente resueltos por Kelsen y en nada se ven modificados por el desarrollo de los DDHH. Luego en el siguiente acápite nos concentraremos específicamente en el Dualismo Derecho Nacional-Derecho Internacional, a la luz de la Norma Fundamental.

Comencemos con el Dualismo Derecho Objetivo-Derecho Subjetivo: en una escueta síntesis, podemos decir que la teoría tradicional sostenía que el Derecho Subjetivo era anterior y superior al Derecho Objetivo. Es decir, podíamos tener Derechos Subjetivos aún cuando el ordenamiento jurídico no los reconociese expresamente (una postura muy similar a la que vimos de los Derechos Morales). Sin embargo, para Kelsen los

4 Pettoruti, Carlos Enrique y Julio César Scatolini. Elementos de Introducción al Derecho. La Ley, Buenos Aires, 2005, páginas 76-77. 
Derechos Subjetivos (en el sentido técnico, que significa la característica de poder ser exigibles), solo pueden ser fruto de su consagración mediante un Derecho Objetivo.

La única aclaración que debemos realizar a esta resolución del Dualismo, es la ubicación normativa del Derecho Objetivo. Esto es, en la teoría kelseniana se hablaba principalmente de una consagración normativa en el ámbito del Derecho Nacional, pero dejaba expresamente abierta la puerta para que el Derecho Internacional fuera regulando cada vez más de manera inmediata las conductas de las personas físicas. Como queda en evidencia del siguiente párrafo de la TPD:

En la misma medida en que el derecho internacional penetra con sus regulaciones en materias que hasta ahora sólo se encontraban normadas por los órdenes jurídicos esta tales particulares, tiene que reforzarse la tendencia al facultamiento y obligación inmediatas de los individuos (Kelsen, 1982: 162) $)^{5}$.

Con lo cual, vemos que existe una indudable compatibilidad entre los DDHH y la TPD en lo que a este Dualismo en particular, ya que sería el Derecho Objetivo (en este caso, los DDHH) quien otorgaría Derechos Subjetivos

Abordemos ahora el Dualismo Persona Física-Persona Jurídica: en otra apretada síntesis, podemos decir que la teoría tradicional sostenía que la Persona Física existía con anterioridad e independencia de la consagración del Derecho Positivo. En cambio, la Persona Jurídica era una creación (ficción) del Derecho Positivo. Kelsen resuelve este Dualismo sosteniendo que existía una confusión entre la Persona Humana y el concepto de Persona del Derecho Positivo. La Persona (para el Derecho), es simplemente un ente de imputación normativa, que puede (o no) condecirse con la idea de Persona Humana. En pocas palabras, para Kelsen es equiparable el concepto de Persona con el de Sujeto de Derecho.

Un claro ejemplo de esta solución brindada por Kelsen la encontramos cuando analizamos el Derecho desde una perspectiva histórica. Muchas veces ha ocurrido que Personas Humanas no tuvieran personalidad jurídica, es decir, no fueran Sujetos de Derecho, como por ejemplo: los esclavos, las mujeres, y así distintos colectivos no eran consideradas Personas en sentido jurídico. Por suerte, y en sentido contrario, vemos cómo en la actualidad se da una extensión del sentido de Persona kelseniano a sujetos que no se condicen con la Persona Humana, como los animales o la Naturaleza (Pacha Mama), a quienes se los identifica como Sujetos de Derecho. Por lo tanto, el concepto de Persona para el Derecho según Kelsen es similar al establecido en el viejo Código de Vélez en su art. 30: "son personas todos los entes susceptibles de adquirir derechos o contraer obligaciones".

Respecto de la compatibilidad de este Dualismo con los DDHH podemos afirmar que no existe una contradicción, ya que los $\mathrm{DDHH}$ de ninguna manera restringen el

5 Podemos ver que en la edición de EUDEBA, el texto resulta muy similar: "En la misma medida en que el derecho internacional penetre en los dominios reservados hasta ahora a los órdenes jurídicos nacionales, reforzará su tendencia a regular directamente la conducta de los individuos", página 162. 
sentido que pueda dársele al concepto de Sujeto de Derecho. Lo que indudablemente ocurre es que se establece un punto de no retorno con respecto a la situación que ha existido con anterioridad en algunos ordenamientos jurídicos nacionales: nunca más podría establecerse la esclavitud o cualquier forma jurídica que niegue el carácter de Sujeto de Derechos a Personas Humanas. Porque su Subjetividad se encuentra ya establecida como necesaria en el Derecho Internacional, siendo este un "coto vedado" (Garzón Valdés), "frontera inviolable" (Bobbio) o "esfera de lo indecidible" (Ferrajoli), es decir: "el conjunto de principios y/o reglas básicas de rango constitucional que ningún poder político constituido, ni siquiera aquél de la mayoría en los Estados democráticos de derecho, puede violar" (Bovero 2008). Como veremos en el siguiente acápite, este concepto resultará esencial a la hora de compatibilizar la Norma Fundamental con los DDHH.

\section{Segundo análisis de compatibilidad: la Grundnorm (Norma Fundamental) y los Derechos Humanos}

A continuación abordaremos sin ningún lugar a dudas la cuestión más dificultosa del presente trabajo, el de la Norma Fundamental (Grundnorm). Lo primero que nos vemos en la obligación de manifestar, es que esta cuestión ha sido vista como el punto débil de la teoría kelseniana, y por lo tanto el argumento más utilizado por sus críticos. Mucho se ha escrito al respecto, y no intenta ser este trabajo un análisis integral de lo que la doctrina ha sostenido acerca de la Norma Fundamental. Simplemente expondremos algunas posturas, para luego proceder a establecer el criterio que utilizaremos para realizar nuestro análisis.

Ya Hart (1968) criticó el sentido hipotético que (supuestamente) le había otorgado Kelsen a la Norma Fundamental, al sostener que "mientras que la validez de otras reglas del sistema puede ser demostrada por referencia a ella, su propia validez no puede ser demostrada, sino que se da por admitida, es postulada o es una hipótesis. Esto, sin embargo, puede ser seriamente equívoco". Hart propone como superadora de la postura de Kelsen a su Regla de Reconocimiento, pero no entraremos aquí en el análisis ni comparación de esta con la Norma Fundamental. También han remarcado el carácter de "hipotética" de la Norma Fundamental Guibourg (2015), Millard (2016) (que la considera una ficción, más que una hipótesis, ya que no puede ser verificada), en el mismo sentido (de ficción) la entendió Cracogna (1998), y Nino la entendió como un "presupuesto epistemológico" (o "hipótesis de trabajo") (Nino, 2003: 121), y en sentidos similares muchos otros, para quienes la Norma Fundamental no era una Norma en sentido estricto.

Una mención aparte requiere el estudio realizado por Candamil Pinzón (2014: 151), quien reelabora una interpretación de la Norma Fundamental, para luego tomarla como "principio fundante" y así extraer de ella "una matriz de modelo de Estado". Si bien no aceptamos todas sus conclusiones y aseveraciones sobre la Norma Fundamental, tomaremos este concepto de Principio Fundante para enlazarlo con la ya mencionada 
teoría del Coto Vedado, para elaborar una teoría propia basada en la interpretación literal de Kelsen.

A continuación tomaremos de manera textual lo dicho por Kelsen respecto de la ubicación de la Norma Fundamental (del Ordenamiento Jurídico Nacional) como una Norma Positiva del Derecho Internacional. Primero en la edición de EUDEBA:

c) La norma fundamental de un derecho nacional es una norma del derecho internacional. Al establecer el principio de que un orden jurídico para ser válido debe tener cierto grado de eficacia, nos limitamos a formular una norma del derecho positivo que no pertenece a este orden jurídico, sino al derecho internacional. A lo largo de esta obra veremos, más detalladamente, que el derecho internacional considera un poder de hecho como legítimo en la medida en que el orden de coacción establecido por dicho poder es realmente eficaz (Kelsen 2003: 116).

Resulta que, si bien algunos autores han tomado el contenido de esta norma para criticar a Kelsen (como por ejemplo Nino ${ }^{6}$ ), no han reparado acerca de la ubicación como Norma Positiva del Derecho Internacional de la Norma Fundamental, lo cual resulta, cuanto menos, extraño. Esto puede deberse sin embargo a que, conforme ya hemos manifestado, esta cuestión fue una de las tantas que sufrió una modificación sustancial para la segunda edición de la TPD. Por ejemplo podemos ver las diferencias cuando sostiene que:

En lo que sigue sólo se considerará, por de pronto, un orden jurídico estatal, es decir, un orden jurídico cuya validez está limitarla a determinado espacio: el llamado territorio del Estado, y que es tenido por soberano, es decir, como no subordinado a ningún orden jurídico superior. El problema del fundamento de validez de las normas de un orden jurídico estatal será explicado inicialmente sin tomar en cuenta un orden internacional considerado como supraordinado o coordinado al orden estatal( Kelsen 2003: 207).

No obstante esta modificación, encontramos luego que plantea la postura contraria:

La situación se modifica, sin embargo, si no se ve en el derecho internacional una parte integrante del orden jurídico estatal, sino un orden jurídico supraordenado a todos los órdenes jurídicos estatales, como un orden jurídico únicamente soberano que delimita a los órdenes estatales en sus respectivos dominios de validez; si no se parte de la primada del orden jurídico de un Estado, sino de la primacía del orden jurídico internacional." Este orden contiene, en los hechos, una norma que representa el fundamento de validez de los órdenes jurídicos de los Estados particulares. De ahí que pueda encontrarse el fundamento de validez de los órdenes jurídicos estatales particulares en el derecho positivo

6 Nino, Carlos Santiago. Introducción al Análisis del Derecho. Editorial Astrea, Buenos Aires, 2003, página 122. 
internacional. Dado que se trata de una norma positiva, y no de una norma meramente presupuesta, la que es fundamento de validez de ese orden jurídico particular (Kelsen 2003: 225).

Como vemos, la contradicción no es total, sino que se plantean distintas posibilidades de fundamentación del ordenamiento jurídico. Sin embargo, poco después establece que:

Puesto que el fundamento de validez de los órdenes jurídicos estatales particulares ya no se encontrará en una norma presupuesta, sino en una norma jurídica positiva, implantada efectivamente, del derecho internacional; se plantea entonces la pregunta acerca del fundamento de validez de esa norma, y, también, la pregunta por el fundamento de validez del orden jurídico internacional, integrado por la norma en que reposa la autoridad del orden jurídico de cada Estado, la norma en que cada orden jurídico encuentra su fundamento de validez inmediato, aunque no último. Este último fundamento de validez sólo puede constituirlo la norma fundante básica del derecho internacional, que, de ese modo, es el fundamento de validez mediato del orden jurídico estatal. En tanto auténtica norma fundante básica, no es una norma impuesta, sino una norma presupuesta (Kelsen 2003: 226)

El tema central de este trabajo se centra en una frase que se encuentra pocas páginas antes de los textos citados, que es la que nos lleva a sostener que debemos adecuar la TPD debido al avance y desarrollo de los DDHH en las últimas décadas. Esa frase sostiene que "El principio de legitimidad está limitado por el principio de efectividad", con el cual Kelsen sostiene que lo esencial de la Norma Fundamental, es que para que se considere legítimo a un ordenamiento jurídico, lo esencial es el acatamiento. He aquí la famosa Pretensión de Coerción que Alexy le achaca (con acierto) a Kelsen.

Nosotros discrepamos con esta aseveración de Kelsen, ya que creemos que la legitimidad del Derecho (tanto Nacional como Internacional) actual, reposa sobre el pilar de la protección de los DDHH. Podríamos reeditar estudios históricos que demuestran que tanto el nacimiento del Constitucionalismo como el del Derecho Internacional (en su configuración actual con Naciones Unidas como eje central) nacieron justamente para brindar protección a los hoy DDHH. Esto probablemente se deba al desarrollo que han tenido los DDHH en las últimas décadas, por lo cual lo entendemos como una reinterpretación de la teoría kelseniana.

Tomando la ya mencionada postura de Candamil Pinzón, entenderemos que en realidad la Norma Fundamental resulta ser un Principio Fundante (del Derecho Internacional y Nacional), que en nuestro caso particular tiene como corolario una Matriz de Estado. Y no es casualidad que nos hayamos centrado en los DDHH, ya que entendemos que los mismos resultan ser uno (aunque no el único) de estos Principios Fundantes, este proveniente directamente del Derecho Internacional, que ha decidido establecer un piso de derechos que garanticen la Dignidad Humana. 
También abrevamos aquí a lo propuesto por Guastini, cuando asegura que "Si se conviene que el principio fundamental del ordenamiento es el criterio de validez, no se comprende cómo ese principio podría ser, él mismo, válido o inválido: los criterios de validez, obviamente, no son válidos ni inválidos". Para luego establecer que "Es válida toda norma que haya sido dictada de conformidad con las metanormas que regulan su producción y que no sea materialmente incompatible con las normas superiores que limitan su posible contenido (Guastini 2011: 367). No abarcaremos en el presente trabajo el concepto de Metanorma por exceder holgadamente el objetivo trazado.

Es decir, entendemos que en el estadío actual del Derecho Argentino, existirían al menos 3 cuestiones que conforman los Principios Fundantes, o "Cotos Vedados" en nuestro ordenamiento jurídico: el Sistema Republicano de Gobierno, la Democracia y los DDHH. Esta postura no se basa solamente en una expresión de deseos, sino que tiene sustento normativo.

Interpretamos al art. 29 de nuestra Constitución Nacional como la muestra de que el Sistema Republicano es uno de los Principios Fundantes, ya que castiga como infames traidores de la Patria a quienes no respeten dicho sistema. El art. 36 nos parece asimismo otra muestra evidente de que la Democracia resulta ser otro de los Principios Fundantes. Y por último, reiteramos la postura de que el propio Sistema Internacional establece que los DDHH resultan ser otro de los Principios Fundantes (no solamente para nuestro ordenamiento jurídico, sino de todo el Sistema).

\section{6- Reflexión final}

Resulta evidente que aún pueden realizarse nuevas interpretaciones de libros clásicos, como la que aquí se propone. Sin embargo, el eje central de este trabajo tiene por eje remarcar la relevancia que tienen los DDHH en nuestro Derecho, especialmente a la luz de los últimos fallos dictados en esta materia por la Corte Suprema (en particular nos referimos a "Muiña" y "Ministerio de Relaciones Exteriores"), que han venido a poner otra vez en duda el rango que ostentan los mismos en nuestro sistema.

Entendemos asimismo que sin ningún lugar a dudas, los DDHH configuran un típico caso de Coto Vedado, siendo su protección un punto de inflexión irreversible en nuestro ordenamiento jurídico. Pero tan solo por si las dudas, y para seguir reforzando su protección, elegimos sostener su carácter de Derecho Positivo como una cuestión política- Compartimos en este sentido lo declarado por Bobbio (1991: 61) (a pesar de disentir acerca de la búsqueda de un fundamento filosófico absoluto de los DDHH, que sí creemos útil): "El problema de fondo relativo a los derechos humanos no es hoy tanto el de justificarlos como el de protegerlos. No es un problema filosófico, sino político". 


\section{Bibliografía}

ALBANESE S. (1999). Los valores en el sistema de derechos humanos. Ed. Ediar

ATIENZA, Manuel (2014). Curso de Argumentación Jurídica. Editorial Trotta S.A., Madrid.

BARBERIS, Mauro. (2013) Los Derechos Humanos como Adquisicón Evolutiva. Doxa, No 36.

BOBBIO, Norberto (1991). El Tiempo de los Derechos. Sistema, Madrid.

(1997) El problema del positivismo jurídico. Versión castellana:

Ernesto Garzón Valdés. Distribuciones Fontamara S.A., México, 1997 (Quinta Edición).

BOVERO, Michelangelo. (2008). Qué No es Decidible. Cinco Regiones del Coto Vedado. Traducción de Álvaro Núñez Vaquero. Doxa, No 31, 2008.

BULYGIN, Eugenio ( 1987) Sobre el Status Ontológico de los Derechos Humanos. Doxa 4.

CANDAMIL PINZÓN, José Gerardo. (2014) De la Grundnorm a la Metanorma Constitucional. Grupo Editorial Ibáñez, Bogotá.

CRACOGNA, Dante. (1998) La Norma Fundamental. Entre la Autocontradicción y la Futilidad. Doxa, No 21- II.

CIURO CALDANI Miguel A (1999)., "Principios y valores en el derecho constitucional" en "Los valores en la Constitución Argentina", ed. Ediar.

DWORKIN, Ronald Los Derechos en Serio. Barcelona, Editorial Planeta, 2015 (tercera reimpresión).

GUASTINI, Riccardo. (2011) Distinguiendo. Traducción de Jordi Ferrer i Beltrán. Editorial Gedisa, Barcelona.

GUIBOURG, Ricardo A. (2015) Derecho, Sistema y Realidad. Editorial Astrea, Buenos Aires.

HART, Herbert L.A.( 1968) . El Concepto de Derecho. Traducción de Genaro R. Carrió. Abeledo Perrot, Buenos Aires.

MILlARD, Éric. (2016) Teoría General del Derecho. Traducción de Bernardo Carvajal Sánchez, Unversidad Externado de Colombia, Bogotá.

NINO, Carlos Santiago. (2007) Derecho Moral y Política II: fundamentos del liberalismo político: derechos humanos y democracia deliberativa. Editorial Gedisa, Buenos Aires.

NINO, Carlos Santiago. (2003) Introducción al Análisis del Derecho. Editorial Astrea, Buenos Aires.

PETTORUTI, Carlos Enrique y Julio César SCATOLINI. (2005) Elementos de Introducción al Derecho. La Ley, Buenos Aires. 
SPECTOR, Horacio. (2001) La Filosofía de los Derechos Humanos. Isonomía No 15, Octubre de.

VERNENGO, Roberto J. (1990) Los Derechos Humanos como Razones Morales Justificatorias. Doxa 7-

\section{Textos Fuente.}

- KELSEN, Hans. (2003) Teoría Pura del Derecho. Traducción de Moisés Nóvile (del francés), EUDEBA, Buenos Aires.

(1982) Traducción de Vernengo. Editorial Andrómeda S.A., Universidad Nacional Autónoma de México, México. 